\title{
A Planar-Chiral Pillar[5]arene-Based Monophosphine Ligand with Induced Chirality at the Biaryl Axis
}

Yuuya Nagata*

Yasuo Shimada

Tsuyoshi Nishikawa

Ryohei Takeda

Makoto Uno

Tomoki Ogoshi*

Michinori Suginome*

Synlett, 2018, 29, 2167

For the final online and print version, $\mathrm{HSICl}_{3}$ was replaced by $\mathrm{HSiCl}_{3}$ in Scheme 1 . 